Check for updates

Cite this: J. Mater. Chem. B, 2021 9. 1259

\title{
Magnetic triggers in biomedical applications - prospects for contact free cell sensing and guidance
}

\author{
Ana F. Almeida, (D) ${ }^{a b}$ Adriana Vinhas, (D) ${ }^{a b}$ Ana I. Gonçalves, (D) ab \\ Margarida S. Miranda, (ID ab Márcia T. Rodrigues (iD *ab and Manuela E. Gomes (ID *ab
}

\begin{abstract}
In recent years, the inputs from magnetically assisted strategies have been contributing to the development of more sensitive screening methods and precise means of diagnosis to overcome existing and emerging treatment challenges. The features of magnetic materials enabling in vivo traceability, specific targeting and space- and time-controlled delivery of nanomedicines have highlighted the resourcefulness of the magnetic toolbox for biomedical applications and theranostic strategies. The breakthroughs in magnetically assisted technologies for contact-free control of cell and tissue fate opens new perspectives to improve healing and instruct regeneration reaching a wide range of diseases and disorders. In this review, the contribution of magnetic nanoparticles (MNPs) will be explored as sophisticated and versatile nanotriggers, evidencing their unique cues to probe and control cell function. As cells detect and engage external magnetic features, these approaches will be overviewed considering molecular engineering and cell programming perspectives as well as cell and tissue targeting modalities. The therapeutic relevance of MNPs will be also emphasized as key components of nanostructured systems to control the release of nanomedicines and in the context of new therapy technologies.
\end{abstract}

Received 19th October 2020

Accepted 18th December 2020

DOI: $10.1039 / \mathrm{d} 0 \mathrm{tb} 02474 \mathrm{k}$

rsc.li/materials-b especially for detection and diagnosis taking advantage of MNPs size, shape, surface charge, surface composition, and contact-free manipulation by a magnetic field (MF).

MNPs have long been studied as contrast agents for magnetic resonance imaging (MRI). ${ }^{2}$ Cells and tissues have negligible magnetic background interference, and MNPs can be used to obtain highly sensitive and quantitative information. Some MNPs formulations have been approved for clinical use as contrast agents for organ imaging (e.g. Ferumoxide), for anemia treatment (e.g. Ferumoxytol) and for thermal ablation (hyperthermia) treatment of tumors (NanoTherm ${ }^{\circledR}$ ). ${ }^{3}$

In recent years, the combination of MNPs with detection, purification and labelling assays led to the development of costeffective and high-resolution biosensing systems. Although these systems were primarily conceived for the analysis and/ or monitoring of specific, sometimes rare, biological analytes (e.g. bioactive molecules, nucleic acids, proteins) ${ }^{4}$ they may also serve as indicators of therapeutic efficacy allowing a realtime non-invasive monitoring in follow-up treatments. Besides the contribution of magnetic detection to the identification, screening and mapping of potential biomarkers, labelling and separation of cellular subpopulations with distinct biological functions can be also performed by magnetic sorting, ${ }^{5,6}$ assisting to the comprehension of biological mechanisms of specific cell subsets and monitoring of cellular events.

\footnotetext{
${ }^{a} 3 B$ 's Research Group, I3Bs - Research Institute on Biomaterials, Biodegradables and Biomimetics, University of Minho, Headquarters of the European Institute of Excellence on Tissue Engineering and Regenerative Medicine, AvePark, Parque de Ciência e Tecnologia, Zona Industrial da Gandra, 4805-017 Barco, Guimarães, Portugal.E-mail:mrodrigues@i3bs.uminho.pt, megomes@i3bs.uminho.pt; Fax: +351 253510 909; Tel: +351 253510 904/913

${ }^{b}$ ICVS/3B's-PT Government Associate Laboratory, Braga/Guimarães, Portugal
} 


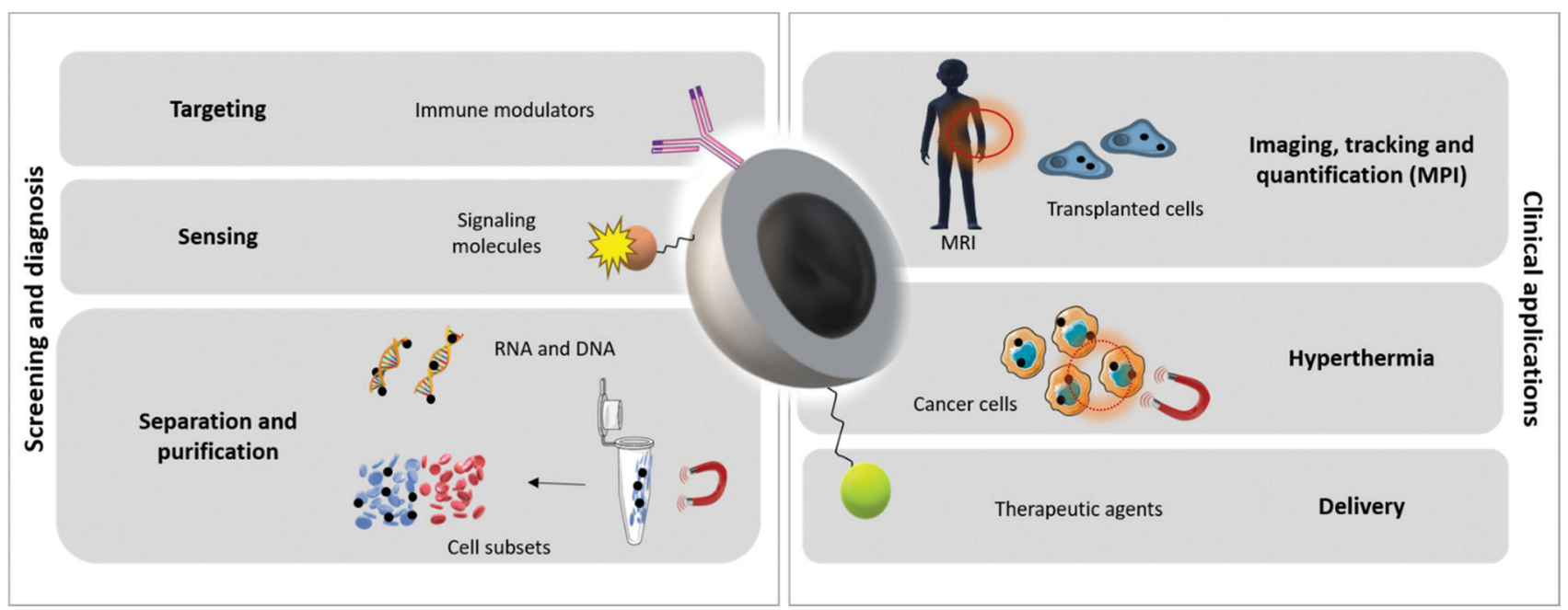

Fig. 1 Schematic representation of current magnetic nanoparticles applications in the biomedical field.

Magnetically assisted therapies using MNPs as highly sensitive and precise nanotools also offer great potential for cell and genebased therapies. ${ }^{7}$ The possibilities for MNPs application in the clinics include cell transfection (magnetofection), DNA enrichment, molecular engineering, and cell programming to probe and manipulate cell function via intracellular regulation, as well as to the treatment of genetic diseases. ${ }^{8}$ As clinical treatments often employ a medicine administration, magneto-controlled delivery systems could efficiently transport the medicine (e.g. drugs, nucleic acids) to the precise location (targeting delivery) with the advantages of reducing the therapeutic dosages, increasing cellular uptake and improving local retention of payloads. ${ }^{9-12}$

The skilfulness and adaptability of MNPs to both diagnosis and treatment modalities makes MNPs advanced theranostic agents, challenging the further development of more sophisticated medicines and their release systems enabling precise and customizable therapeutics adding value to magnetic based technologies in healthcare.

The breakthroughs in magnetically assisted technologies for contact-free control of cell and tissue fate opens new perspectives to improve healing and instruct regeneration reaching a wide range of disorders. Interestingly, several studies have reported a beneficial effect of magnetic fields in meliorating inflammatory signs and to assist healing ${ }^{13}$ as well as the exciting role of MNPs in monitoring inflammation in vivo. ${ }^{14}$

Although inflammation is a critical step for healing to occur, persistent inflammatory cues hindering healing may lead to chronic (persistent) inflammation stages with detrimental effects over the tissues and organs. ${ }^{15}$

Inflammatory conditions are typically controlled with systemic and local administration of anti-inflammatory drugs, namely nonsteroidal anti-inflammatory drugs (NSAIDs) and corticosteroids that either stop or prevent the inflammation to be resolved, ultimately compromising healing outcomes and the functional recovery of tissues or organs. Furthermore, prolonged used of such drugs has been associated with adverse side effects, including ischemia and myocardial infarction, impaired wound healing, osteoporosis, or peptic ulcers. ${ }^{16,17}$ The unsuitable clinical response to address abnormal inflammatory cues demands for alternative therapeutic interventions that could be provided by magnetically assisted strategies in supporting the modulation, guidance and monitoring of inflammatory events, influencing immune cell responses and contributing to proper healing solutions, holding promise to improve patients wellbeing and quality of life.

\section{Opportunities for magnetically assisted technologies to improve healing and instruct regeneration}

Healing is a necessary and essential process for the restoration of physiological balance after disease or damage in living tissues, organs, or biological systems with concomitant recovery of function. It is a highly orchestrated process, and usually characterized by three overlapping phases: inflammation, proliferation, and remodelling. ${ }^{18}$ Immediately after injury, platelet activation increases vascular permeability and the release of chemokines. Chemokines stimulate resident immune cells and promote the recruitment of circulating inflammatory cells to the injury site. Neutrophils appear within minutes of the initial injury and are followed shortly by recruited macrophages. After inflammation, proliferation takes over and an increase in fibroblast activity gives rise to the deposition of a provisional extracellular matrix (ECM), mainly composed by collagen. At this stage new blood vessels are also formed. Finally, during remodelling, the provisional ECM matures, and collagenous fibers are organized to improve and restore the mechanical and biochemical properties of the tissues. ${ }^{18}$

Inflammation is a signal-mediated response working together with the immune system. Persistent or chronic inflammation has been associated to almost every chronic disease, including arthritis, cardiovascular disease, and cancer. The balance between a beneficial inflammatory phase to boost tissue repair 
and the resolution of the inflammation for the continuum of the healing stages dictates proper healing versus chronic conditions. The fine-tuning regulation of inflammation challenges cuttingedge methods with more sensitive and predictive power for screening and diagnosis of biomarkers with therapeutic value, for specific tissue guidance, and for immune modulatory therapies.

Magnetic stimulus has been shown to improve inflammatory signs; alleviating pain, assist bone fracture healing ${ }^{13}$ and influence cytokine release kinetics. ${ }^{19,20}$ Magnetic stimuli can be provided by static-(SMF), alternating-(AMF), and pulsed electro- magnetic fields (PEMF). SMF are commonly provided by a permanent magnet which generates a MF that does not change its direction over time. In contrast, PEMF and AMF are produced by alternating currents that change their direction over time with a specific frequency. Additionally, PEMF treatments are applied in pulses instead of a constant exposure to the electromagnetic field.

Reported studies show that magnetic stimuli influence cellmembrane processes such as the membrane potential, ${ }^{21}$ ionic channels $^{21-24}$ or cell receptors. ${ }^{25,26}$ Moreover, magnetic stimulus was also described to influence the response of several types of cells, including immune cells, ${ }^{20,21}$ cells from musculoskeletal tissues, ${ }^{27}$ and nervous system, ${ }^{28}$ suggesting an intercellular networked action to control abnormal inflammatory cues in nearby tissues.

Macrophages are key players in inflammatory mechanisms and a source of proteases, cytokines, growth factors (GF) and ECM components. These cells can regulate their phenotype to perform specific functions accordingly to environmental factors. In response to different signals, macrophages may undergo to a classically M1 activation stimulated by toll-like receptors (TLR) ligands, IFN- $\gamma$, or alternatively M2 activation stimulated by IL-4/IL-13. ${ }^{29} \mathrm{M} 1$ phenotype is mainly activated by the innate immune system and are traditionally associated with a pro-inflammatory response. ${ }^{30}$ Conversely, M2 phenotype is associated with resolution of inflammation and structural tissue healing. ${ }^{30}$ Several studies point that macrophages sense magnetic stimuli and respond towards M2 phenotype. $^{20,21}$ This adaptation was shown by an elongated shape which led to an M2-specific gene pattern. ${ }^{21}$ A SMF influenced the ionic currents on the macrophages membrane disrupting $\mathrm{Ca}^{2+}$ homeostasis inducing changes in actin cytoskeleton and, subsequently affecting polarization. ${ }^{21}$ Our group also demonstrated that low frequency PEMF induced a M2 phenotype in co-cultures systems with tendon cells exposed to an induced pro-inflammatory environment. $^{20}$

Magnetic stimulus has also been reported to drive ECM remodelling supporting collagen fiber alignment in artificial matrices, ${ }^{31}$ and to provide topographical and chemical cues to cells laden in 3D scaffolds. These features can assist the in vitro recreation of the ECM organization and architecture for real time visualization of single cell dynamics or multicellular assembly. $^{32}$

The understanding of magnetic perception by cells and tissues could assist the regulation of complex processes as inflammation, controlling and resolving inflammatory events and contributing to the management of diseases and pathologies using less invasive and high-fidelity approaches. Such approaches could be complemented with magnetic elements as MNPs, whose design can be accurately controlled to meet cellular requirements.

\section{MNPs for biomedical strategies}

With the increasing developments of nanotechnology, several nanomaterials emerged promptly with unique physical, chemical, and biological properties. Among the nanomaterials, MNPs include metals (e.g. iron, cobalt, nickel), metal oxides (e.g. magnetite $\left(\mathrm{Fe}_{3} \mathrm{O}_{4}\right)$ ), alloys (e.g. iron-carbon, iron-platinum, neodymium-iron-boron), and ferrites $\left(\mathrm{ZnFe}_{2} \mathrm{O}_{4}, \mathrm{CoFe}_{2} \mathrm{O}_{4}\right.$, $\mathrm{BaFe}_{12} \mathrm{O}_{19}$ ) that have aided the efficient development of modern technology from magnetic separation, catalysis, environmental treatment to biomedicine. ${ }^{33}$ Although cobalt and nickel nanoparticles and derivatives have shown excellent magnetic properties, they possess high toxicity which could compromise the safety and in vivo applicability. Conversely, superparamagnetic iron oxide nanoparticles (SPIONs) combine unique magnetic properties with biocompatibility, no immunogenicity, and low toxicity making SPIONs excellent candidates for biomedical applications. Also, SPIONs can be degraded and cleared from the body via endogenous metabolic iron pathways and main routes for excretion, respectively. ${ }^{34,35}$

SPIONs consist of a core made of iron oxide (magnetite $\left(\mathrm{Fe}_{3} \mathrm{O}_{4}\right)$ or maghemite $\left.\left(\gamma-\mathrm{Fe}_{2} \mathrm{O}_{3}\right)\right)$. When the size of the SPIONs is below $20 \mathrm{~nm}$ they show a superparamagnetic behaviour that is, they are characterized by a high magnetic moment when exposed to a MF and have no magnetic moment when the MF is removed, ${ }^{36}$ thus reducing the potential for aggregation.

In general, the synthesis of SPIONs is a multistep procedure, which affects their physico-chemical properties. Synthesis of SPIONs traditionally includes top-down methods and wetchemistry methods. Top-down methods are based on physical and mechanical techniques to transform bulk phase materials into SPIONs. Laser ablation, ultrasonication, microwave irradiation or ball milling are commonly used in the industry as "green" methods to obtain very high yield using automation and reproducibility processes. However, the lack of control in the size distribution and shape of the SPIONs compromises their applicability in precision biomedicine. Wet chemistry methods constitute the conventional approaches to produce SPIONs for biomedical applications (Table 1). Clinically approved SPIONs used as MRI contrast agents are produced by co-precipitation using mild reaction conditions with excellent control over SPIONs shape despite the poor size control.

More recently, novel synthesis techniques have been developed such as microfluidic and biogenic synthesis (Table 1). Microfluidics enables the production of SPIONs with controlled size, shape, and morphology in a faster, continuous, and automated process with lower energy consumption. High quality SPIONs can also be produced from living organisms as magnetotactic bacteria and fungi through biogenic synthesis. This non-toxic, eco-friendly method takes advantage of naturally occurring biomolecules (e.g. nanometer-sized crystals of magnetite) and the biological machinery (e.g. biomineralization and magnetite nucleation proteins) to produce SPIONs with narrow size distribution. 
Table 1 Overview of MNPs synthesis methods for biomedicine $e^{37-41}$

\begin{tabular}{|c|c|c|c|}
\hline & Types & Advantages & Disadvantages \\
\hline Wet chemistry route & $\begin{array}{l}\text { Non-hydrolitic (organic media) } \\
\text { - Thermal decomposition } \\
\text { - Microwave assisted synthesis }\end{array}$ & $\begin{array}{l}\text { Excellent control over size } \\
\text { Highly crystallinity }\end{array}$ & $\begin{array}{l}\text { Very time consuming } \\
\text { Requires a water phase transfer }\end{array}$ \\
\hline Microfluidics & $\begin{array}{l}\text { Single-phase flow (continuous flow) } \\
\text { - Capillary tube microreactors }\end{array}$ & $\begin{array}{l}\text { Simple and highly flexible } \\
\text { Facile in operation }\end{array}$ & $\begin{array}{l}\text { Adhesion to the channel and clogging } \\
\text { Wide residence time distribution } \\
\text { Large size distribution }\end{array}$ \\
\hline Biogenic synthesis & $\begin{array}{l}\text { mediated by } \\
\text { - Bacteria } \\
\text { - Fungi } \\
\text { - Plants } \\
\text { - Proteins associated to biomineralization }\end{array}$ & $\begin{array}{l}\text { Narrow size distribution } \\
\text { Very low energy consumption } \\
\text { "Green" method }\end{array}$ & $\begin{array}{l}\text { Poor reproducibility } \\
\text { Low yield } \\
\text { Difficult to scale up } \\
\text { Poor automation }\end{array}$ \\
\hline
\end{tabular}

\section{The role of MNPs properties in biological interactions}

The size, shape, charge, and surface chemistry of MNPs need to be finely adjusted since these properties can affect MNPs interactions in the body, including cellular uptake, in vivo fate, and toxicity (Fig. 2).

The particle size and shape are important aspects for cell interactions and whether MNPs are internalised by endocytosis

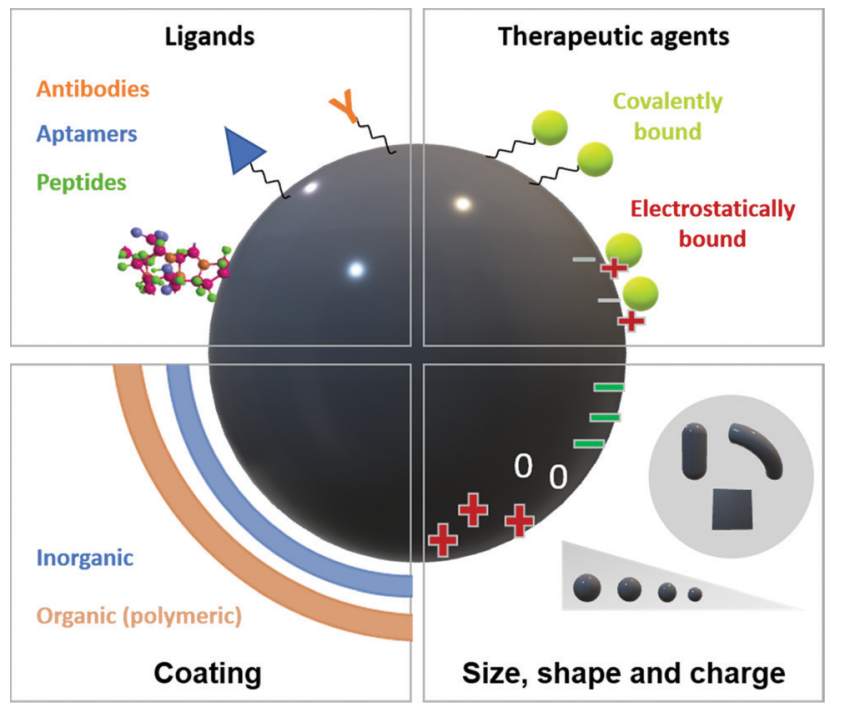

Fig. 2 The physico-chemical properties of magnetic nanoparticles with influence on cell responses, including biodistribution, biodegradability, toxicity, and clearance from the body. or delivered by magnetofection, directly passing through the cellular membranes.

The shape of MNPs also contributes for different cell responses. In a study by Mahmoudi et al. cells interacting with nanobead-, nanoworm-, and nanosphere-shaped SPIONs show increased toxicity compared with cells in contact with nanorods and colloidal nanocrystal clusters. ${ }^{42}$

Particle charge is intrinsically related to the surface chemistry and is another important design characteristic. Positively charged or neutral particles will favour cell membrane interactions, which are naturally negative, and will assist cell internalization processes or targeting receptors. To improve chemical and colloidal stability, biocompatibility, and the functionalization/conjugation, MNPs are often coated with organic and inorganic materials. Organic coatings include small molecules, surfactants, dendrimers, macrocycles, natural and synthetic polymers, while the inorganic materials typically refer to gold, silver, or silica. For example, in an interesting approach SPIONs have been surface functionalized with the macrocycle cucurbit[7]uril and the drugs were noncovalently conjugated by supramolecular host-guest chemistry allowing their release on demand. ${ }^{43,44}$

The targeting efficiency to cells/tissues ${ }^{45}$ is often improved through the formation of MNP-conjugates with drugs, bioactive molecules, fluorescent markers or targeting ligands (e.g. small molecules, antibodies, aptamers, and peptides) (Fig. 2). Moreover, ligands such as polyethylene glycol (PEG) can be conjugated to prevent protein adsorption and improve blood circulation. $^{46}$ 
The precise contribution of each physico-chemical property of the MNPs to the successful design and purpose in cell responses is still controversial. Although the coating can directly influence MNPs interactions with living cells and tissues, some studies refer that charge cues may be more relevant than the nature of MNPs coating in biological responses. PEG-SPIONs with neutral charge were found to be internalized less readily by the reticuloendothelia system (RES) and showed a lower uptake by the liver when compared to negatively-charged coated SPIONs (both PEG and carboxydextran). ${ }^{14}$

SPIONs larger than $100 \mathrm{~nm}$ have been reported to be quickly trapped in the liver and spleen through macrophage phagocytosis, whereas SPIONs smaller than $10 \mathrm{~nm}$ are expected to be eliminated through renal clearance. ${ }^{47}$ SPIONs ranging from 10 to $100 \mathrm{~nm}$ are thought to benefit from slower opsonization and clearance from the RES. ${ }^{47}$ Despite the tendency for MNPs bioaccumulation in some organs, several studies reported evidences of the biodegradation and clearance 2 weeks after in vivo administration. ${ }^{47}$ As such, it is imperative to design MNPs and scrutinize their properties according to their goal application to identify possible bioaccumulation, confirm MNPs biodegradability and nontoxicity, and to assure their clearance from the body.

In recent years, hybrid materials integrating MNPs as magnetic polymeric nanocomposites, magnetic liposomes, magnetic micelles and magnetosomes, have also been developed with the aim to combine advantages of both systems and enable multiple modalities in a unique system. These hybrid materials are generating particular interest in the development of nanocarriers for both diagnosis and therapeutic applications due to the advantages relative to conventional MNPs such as higher magnetic moment, higher MRI sensitivity and higher drug loading capacity. ${ }^{48,49}$

\section{Current and prospective medical applications of SPIONs}

i. In imaging technologies. Imaging technologies based on SPIONs enable non-invasive visualization of cells/tissues and have shown potential for inflammation diagnosis and monitoring treatment response.

Several SPIONs formulations developed for MRI allow the non-invasively visualization and monitoring of infection and inflammation, thereby assisting an earlier intervention in inflammation-associated diseases and better outcomes. The accumulation of SPIONs phagocytosed by macrophages or via autophagy mechanisms can enable the MRI detection of macrophage infiltration. The same approach has been investigated for tracking and imaging of transplanted cells. ${ }^{50}$ Particular subsets of macrophages, in the case $\mathrm{CD}_{206^{+}} \mathrm{M} 2$-like macrophages, can be explicitly observed in vivo using antibiofouling mannose SPIONs. ${ }^{51}$ As macrophages tend to concentrate in inflamed tissues, the design of molecular imaging probes to target specific surface cell molecules, such as CD206, may accomplish to distinguish between different physiological states of disease or healing.

Advances in imaging techniques, such as the magnetic particle imaging (MPI), point new developments for ex vivo magnetic cell labelling and in vivo cell tracking using SPIONs as tracers. MPI is an emerging non-invasive 3D real-time tomography method that detects and quantifies SPION tracers at spatial resolutions comparable to established modalities. In the particular case of inflammation monitoring, SPION tracers may be captured/internalized by phagocytic cells at the site of inflammation or can be loaded/incorporated into phagocytic cells and tracked as they migrate and accumulate in inflammation regions. $^{52}$

Although MPI is still at the infancy, it is rapidly gaining interest showing incremental steps toward medical purposes requiring fast dynamic information.

Imaging cerebral vasculature and perfusion was already achieved by MPI in a murine model of ischemic stroke with high sensitivity and high temporal resolution. ${ }^{53}$ Approaching a clinical context, a human sized MPI system was developed for monitoring the neurovascular status of patients with high sensitivity (14.7 $\mathrm{ng} \mathrm{mL} \mathrm{m}^{-1}$ corresponding to $\left.2 \mu \mathrm{g} \mathrm{Fe}\right) .{ }^{54}$ The system detected and monitored cerebral perfusion in ischemic stroke and could be applied to monitor the follow up of patients with a high risk of restenosis or of haemorrhage. The small size of the MPI equipment and its set up as a mobile unit allows the diagnosis at the patient's bed rather than bringing the patient to the system. The early detection of cerebrovascular diseases and incidents through MPI may lead to an earlier therapy decision-making and contribute to reduced patient mortality and morbidity.

ii. In molecular and cellular technologies. Magnetic particles (MPs) in the nanometer to micrometer size range have been explored for biosensing applications in three different approaches: (1) for analyte concentration and separation; (2) to amplify the signal and (3) to contribute to the signal readout depending on their magnetic, optical, and electrical properties. ${ }^{55}$

A microfluidic-on-chip device based on a multilaminar flow platform was developed for the quantitative detection of the inflammatory biomarker C-reactive protein (CRP) in human serum samples. ${ }^{56}$ In this sandwich immunoassay, functionalized MPs were pulled, by an external magnet, through alternating streams of reagents and washing buffers. The great advantage of this platform was the speed of the assay, which was less than $60 \mathrm{~s}$ and represented a significant improvement over the $50 \mathrm{~min}$ necessary for off-chip MP-based immunoassays and the $300 \mathrm{~min}$ required for ELISA assays. To increase the signal of soluble inflammatory mediators in body fluids, MPs covered with antibody against TNF $\alpha$ were used to measure TNF $\alpha$ with lower detection limits than the ones achieved using a non-magnetic sensor. ${ }^{57}$ Other sensing strategies used an alloy FeCo nanoparticle based platform and a giant magnetoresistive sensor for improving signal information enabling the quantification of IL-6, an inflammatory cytokine and a potential cancer biomarker, in human serum samples. ${ }^{58}$

MNPs-based biosensing technologies can also be applied to nucleic acid detection and identification, and potentially replace the current gold standard approach which is polymerase chain reaction (PCR). Although highly reliable, PCR is a timeconsuming and labour-intensive technique, while MNPs based systems may represent faster, low-cost, and more sensitive tools. 
In order to overcome PCR limitations, Pang et al. proposed a functionalized $\mathrm{Fe}_{3} \mathrm{O}_{4} @ \mathrm{Ag}$ MNPs biosensor to capture and ultrasensitively detect microRNA from total RNA extracts avoiding PCR pre-amplification treatment. ${ }^{59}$ Similarly, Wang and coworkers developed a simple method using silica coated MNPs to simultaneously extract DNA and RNA from the same sample offering high quality and quantity of nucleic acids suitable for downstream activities of PCR and RT-PCR. ${ }^{60}$

Besides MNPs potential for screening and diagnostic, MNPs can improve highly sensitive and efficient techniques as PCR, increasing the specificity and predictive value of the biological data for clinical approaches.

\section{Magnetically assisted targeted strategies}

The lack of accessibility of many diagnostic and therapeutic agents to pathological niches remains a clinical challenge. Targeting strategies enable a direct delivery of precise and informative signals to specific cell types, cell receptors or other finely regulated molecules available at the cell membrane to guide desirable cell responses.

\section{Magnetic cell targeting}

Cell targeting by means of magnetic triggers has evolved in the last decade as a method to improve either the delivery of cargos to cells or the retention of therapeutic cells near a target organ.

The first challenge in targeting strategies is the target itself. Biomolecules, typically peptides, proteins, protein complexes (e.g. cell receptors) or structural elements of the cell membrane (e.g. glycoproteins or cholesterol), should enable to identify activated cells, tissues, or pathophysiological states for precise cell and tissue guidance to achieve effective therapeutic outcomes.

i. Macrophage targeting. The innate system is extremely important to tissue injury response and represents the first line of defense against pathogens. Diseased or injured tissues often accumulate macrophages derived from circulating blood monocytes, which can bring valuable information about both pathology and healing progression. As professional phagocytic cells, macrophages can control immune responses at different levels. ${ }^{15}$ While the uptake of pathogens leads to the presentation of associated antigens triggering an inflammatory response against the pathogen, macrophages also uptake apoptotic bodies during tissue remodelling which promotes the secretion of antiinflammatory cytokines and maintenance of homeostasis. Given macrophage capability to control the outcomes of an immune response, these cells are common targets for the treatment/ diagnosis of a wide range of diseases. ${ }^{61}$ Vascular inflammation is a key contributor for the progression of vascular diseases such as atherosclerosis. Atherosclerotic macrophages highly express $\alpha_{v} \beta_{3}$ integrin, a cell surface glycoprotein receptor, with affinity for Arg-Gly-Asp (RGD) binding. RGD-conjugated human ferritin SPIONs were investigated as a detection mean of macrophages infiltration using in vivo MRI to assess vascular inflammation in murine carotid arteries and abdominal aortic aneurysm. ${ }^{62}$

The coupling of SPIONs with CD86 or CD206 antibodies targeting M1 and M2 macrophages subsets, respectively, has revealed the importance for early and better diagnosis and therapy of chronic obstructive pulmonary disease using noninvasive imaging. ${ }^{63}$ Although these approaches are oriented to detect and identify disease/injured regions in the body with active sites of inflammation, magnetically guided cell delivery systems can also be valuable to program cells involved in crucial events for disease progression such as inflammation, fibrosis and impaired healing. In this sense, magnetic cationic liposomes were developed for the simultaneous introduction of SPIONs and plasmid DNA (pDNA) into macrophages to enable both the magnetization of macrophages and the regulation of their function. ${ }^{64}$ Less conventional approaches for controlling macrophages adhesion and subsequent polarization were pursued using a magnetic remotely controlled magnetic nanocage conjugated to a RGD-bearing gold via a flexible linker. ${ }^{65}$ The uncaging of RGD temporally encouraged macrophages adhesion and subsequent M2 phenotype while inhibiting their M1 phenotype both in vitro and in vivo.

ii. Magnetic mechanosensing and activation of cells. The perception over different external stimuli by cells and their interpretation is a signalling process called mechanotransduction. ${ }^{66}$ The timescale of these events, which include stimulus, sensing, signalling, gene expression, and cell function, ranges from seconds for the stimulation of mechanosensors, hours for altered gene expression, days for changes in cell behaviour and function, and weeks for tissue development. ${ }^{67}$ Within mechanobiology, novel nanotechnology tools might have the potential to decipher molecular mechanisms in the modulation of signalling cascades underlying healing and repair. ${ }^{68}$ In this sense, the MF promotes mechanical motion of the MNPs, which in turn can stimulate the cells, subcellular structures and biomacromolecules to which MNPs are attached. This approach has been defined as magnetic mechano-activation to remotely deliver mechanical stimulation directly to individual cells. ${ }^{69}$ In this way, MNPs attached to the cell membrane can be manipulated using an oscillating gradient of EMF, applying forces in the picoNewton range to the particles and activating membrane receptors or ion channels. ${ }^{70,71}$

In the last decade, pioneering works have used magnetic mechano-actuation and MNPs technology to open ion channels or target cell membrane receptors, mediating mechanotransduction of stem cells and regulating signalling cascades. ${ }^{22-24,26,72}$ These constitute successful examples of magnetically targeted strategies that have been explored in the osteogenic and tenogenic differentiation of stem cells aiming at tissue regeneration.

\section{Magnetically responsive systems to control the release of nanomedicines and regulatory cargos}

SPIONs based systems offer substantial hopes to nanomedicine and to the drug delivery field since the synergetic combination with magnetic fields, enables a space- and time-controlled delivery. As nanocarrier systems, the protection of payloads from both metabolism and off-target effects is assured as well as the control over the release and specific delivery of cargo to cells.

Therapeutic cargo can be conjugated to MNPs by electrostatic interactions, covalent bonds and adsorption or incorporated in hybrid MNPs carrier systems. By application of an EMF gradient, 
these systems are guided and concentrated at the desired location thus providing a specific and localized cargo delivery. Ketorolac, a NSAID widely prescribed for relieving pain and controlling inflammation, was conjugated to SPIONs and intrathecally administered to mice in a chronic inflammatory pain model. ${ }^{73}$ A polarity modulated MF was used to guide and retain the ketorolac-SPIONs at the lesion site and significantly promoted an analgesia effect with suppression of cyclooxygenases. ${ }^{73}$ The local delivery of therapeutic cargo by MNPs carrier systems has the potential to increase treatment efficiency and minimize side effects associated to systemic routes and to the administration of nanomedicine overdosages.

i. Magnetic SPIONs-drug systems. In the presence of high frequency AMFs, SPIONs generate heat and can trigger the local release of drugs from thermally sensitive materials in association with hyperthermia, which is typically used for cancer treatment. Conversely, the exposure to low frequency AMF, leads to a negligible heat effect, and the SPIONs agitation in the systems and their mechanical deformation allows a controlled release of the cargo. However, low frequency AMF is mainly used in laboratory conditions, while current magnetic therapies are typically applied as PEMF and used for controlling and treating inflammation.

The SPIONs motion produced by the PEMF led to the increase of the bilayer permeability of magnetoliposomes resulting in an on-demand release of a hydrophilic model drug. ${ }^{74}$ The combination of SPIONs based carriers and PEMFs is a promising dual approach, as synergistic effects of both modalities could be effectively utilized for remotely activated drug delivery and for resolving inflammation.

ii. SPIONs based systems for nucleic acid therapies. Nucleic acid (NA) therapies rely on NA molecules or closely related chemical compounds as intracellular tools to inhibit the function of a specific gene to treat or prevent diseases. However, the transport of NA into the cell is challenged by poor cellular uptake, inefficient endosomal escape, fast degradation in blood and renal clearance.

Delivery systems based on MNPs/NA magnetic complexes offer opportunities to overcome transport limitations and to challenge the design of more effective therapies. MNPs present an enhanced gene transfer efficiency which is a significant advantage relative to other delivery systems. In MNPs based systems, the cationic MNPs bind electrostatically to negatively charged phosphate backbone of genetic material forming magnetic complexes. The MNPs/NA complexes are then transfected into cells under the guidance of a MF (magnetofection).

Functional RNA molecules combined with polyethylenimine (PEI)-coated SPIONs have demonstrated improved efficacy of transfection in vitro, compared to the use of polycations or lipids alone, ${ }^{7}$ and specific gene (EGFP) silencing efficiencies in human breast cancer cells. ${ }^{7}$ Not only SPIONs-RNA showed an efficient knockdown of target protein expression but also SPIONs may efficiently protect nucleic acids from nucleases degradation and deliver sensitive molecules to human cells. Although PEI-NA complexes, have been widely used for NA delivery taking advantage of the high transfection efficiency by PEI, some studies reported high cytotoxicity in the presence of high molecular PEI. ${ }^{75}$ Thus, MNPs-RNA complexes without $\mathrm{PEI}^{76}$ could be an alternative approach to improve the biological interactions of such systems.

The recent gene editing advances by clustered regularly interspaced short palindromic repeats-associated protein (CRISPRCas9)-based technology have been restrained by the still limited control of the precise activation of CRISPR-Cas9 in the target tissue. One of the most popular modes of delivery for genetic material including DNA sequences encoding CRISPR/Cas9 is the use of viral vectors. In a study by Zhu et al., SPIONs were combined with recombinant baculoviral vectors (BVs) to improve the local activation of CRISPR-Cas9 mediated genome editing upon the application of a MF. ${ }^{77}$ Not only SPIONs improved cell entry and transduction efficiency of BVs in vitro as it helped overcome serum inactivation of BVs. Despite their high transduction efficiency, viral vectors have some drawbacks such as increased immunogenicity, expensive large-scale production, and the risk of integrating viral sequences into the target genome. ${ }^{78}$ From this viewpoint, non-viral delivery systems for CRISPR/Cas9 are a promising strategy and MNPs can be allies to overcome these limitations. Recently, PEI-SPIONs combined with a gradient MF were shown to improve efficiency of DreamFect Gold, a commonly used transfection reagent, to deliver CRISPR/Cas9 constructs in porcine fetal fibroblasts for generating genetically modified pig models. ${ }^{79}$ Using another approach, Rohiwal and colleagues generated SPIONs by co-precipitation and complexed with CRISPR/Cas9 to deliver genetic material into cells without the help of transfection reagents. They showed that CRISPR/ Cas9-PEI-SPIONs complexes were internalized by cells via endocytosis due to the cationic PEI, which triggered endosomal disruption and caused the release of the complexes into the cytoplasm. ${ }^{80}$ This strategy showed promising outcomes for efficient therapeutic genome editing in both in vitro and in vivo modalities.

In sum, MNPs-NA systems can improve safety and utility of gene editing tools presenting exciting opportunities to overcome the challenges of DNA/RNA-based therapeutics and to contribute to the cure or prevent the development of several diseases by altering the expression of endogenous genes in a more controllable manner.

\section{Advancing SPIONs based strategies into tissue engineering and regenerative medicine}

A key feature of MNPs based systems in line with tissue engineering and regenerative medicine (TERM) is their potential contribution for repair of damaged tissues and organs (Fig. 3). MNPs and magnetic fields have gained visibility in numerous domains of TERM as MNPs can integrate sophisticated delivery systems of $\mathrm{GFs}^{81}$ and other bioactive molecules. ${ }^{12}$ Moreover, they can act as extra and intra-cellular triggers ${ }^{20,82}$ modulating cell behaviour to improve the regenerative potential at the injury site, and guide the maturation of the tissue engineered construct in scaffolding strategies. ${ }^{83-85}$

i. Magnetic patches to improve healing using cell sheet technology. Magnetic cell sheets combining cells and MNPs under the actuation of an EMF are an example of a magnetically controlled structure of contiguous ECM and intact cell-cell junctions, 


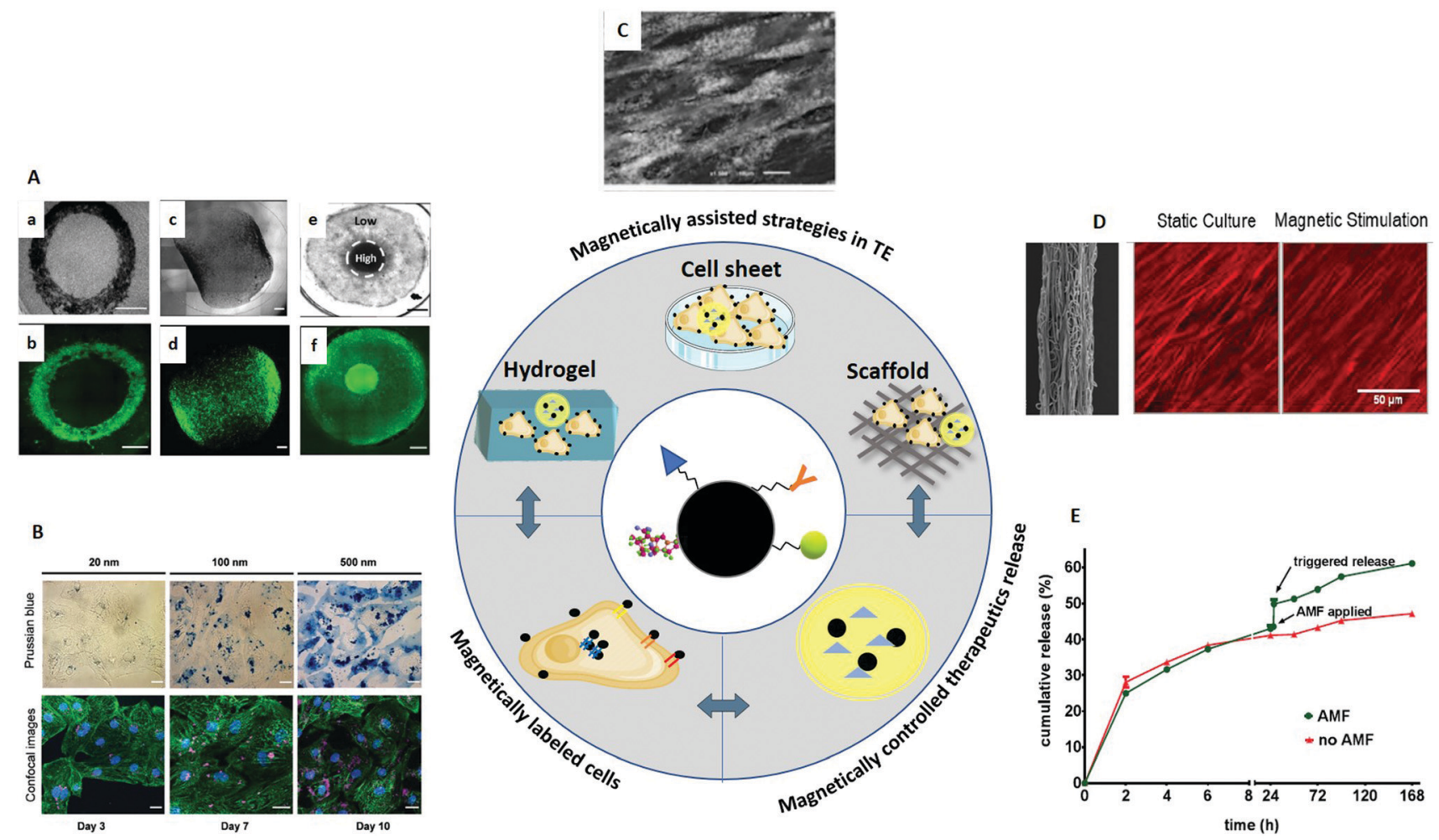

Fig. 3 Regeneration of tissues and organs with magnetically assisted strategies. (A) Engineering of patterned 3D human cardiac tissue constructs combining magnetically-labelled human induced pluripotent-stem-cell-derived cardiomyocytes (hiPSC-CMs) and collagen hydrogels created by magnetic field manipulation; (a-f) representative bright-field (top) and fluorescence (bottom) images of the patterned tissues in response to different magnetic field configurations: ( $a$ and b) ring magnet, ( $c$ and d) parallel magnets, and (e and f) circular magnet used to tailor different microstructures of cardiac tissues (adapted from Zwi-Dantsis et al., 2020). (B) Targeting and magnetic labeling of hiPSC-CMs for incorporation in collagen hydrogels to engineer patterned 3D human cardiac tissue constructs; Prussian blue staining (top images) and confocal fluorescence (bottom images) of the treated CMs $24 \mathrm{~h}$ after labeling with SIRPA-MNPs of different magnetic core sizes: $20 \mathrm{~nm}, 100 \mathrm{~nm}$, and $500 \mathrm{~nm}$ (adapted from Zwi-Dantsis et al., 2020). (C) Scanning electron microscopy (SEM) micrographs of magnetic cell sheets (magCSs) of human adipose-derived stem cells (hASCs) cultured for 7 days in alpha-MEM culture (iron oxide MNPs in bright white) (adapted from A. Gonçalves et al., 2017). (D) SEM image of polycaprolactone/hybrid magnetic nanoparticles (PCL/DT NP-5) threads (left image) and confocal microscopy images of the cytoskeleton (red: phalloidin) of hASCs cultured on PCL/DT NP-5 scaffolds for 21 days. hASCs tend to align under magnetic stimulation (adapted from A. Tomás et al., 2019 with permission from The Royal Society of Chemistry). (E) Cumulative drug "P3" release profile from the magnetic particles after 7 days upon an alternated magnetic field (AMF) of $1000 \mathrm{~Hz}, 3 \mathrm{mT}$ (green line), showing the contribution of magnetic actuation for controlled drug release. Red line represents non-stimulated group (reproduced from M. Miranda et al., 2018).

offering effective means to stimulate and assess the fate/distribution of transplanted constructs in a non-invasive manner. ${ }^{86}$

Magnetic force-based tissue engineering was first termed in 2004 in the construction of keratinocyte sheets with magnetic cationic liposomes. ${ }^{87}$ This methodology emerged as a way of creating cell sheets responsive to magnetic force which is applied to manipulate their attachment and harvest from culture. Magnetic cell sheet technology has shown positive results in vascular, ${ }^{88-90}$ musculoskeletal, ${ }^{82,91-93}$ and cardiac ${ }^{94}$ tissue engineering (TE) strategies, however, current literature provides very few examples of magnetic cell sheets in the context of inflammation resolving therapies. Our group has previously reported the development of cell sheets that exhibited a tendon-like ECM, demonstrating good mechanoelastic properties and magnetic responsiveness, thus suggesting the applicability of these scaffold-free patches toward tendon therapies. ${ }^{82}$

The exogenous delivery of stem cells labelled with SPIONs assisted by MF stimuli may also trigger the inflammation resolution by modulating the inflammatory environment in situ. ${ }^{27,95}$
Recently, GF-immobilized cell sheets were reported by Zhang and co-workers ${ }^{96}$ in which SPIONs were coated with nanoscale

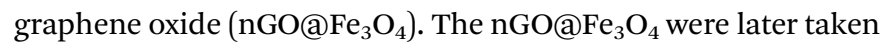
up by dental-pulp stem cells (DPSCs) driven by endocytosis, and accurately controlled by magnetic-force-mediated deposition. BMP2 and TGF $\beta 3$ induction factors were sequentially immobilized into the cell sheet enabled by the graphene oxide coating forming an integrated osteochondral complex. One week after subcutaneous implantation in nude mice, DPSCs were shown to differentiate into chondrocytes and osteoblasts exhibiting potential for osteochondral regeneration. ${ }^{96}$

Bone marrow-derived mesenchymal stem cell sheets labelled with both pkH67 green fluorescent and SPION tracers were developed by Rahmi et al. ${ }^{97}$ and applied in a mouse fistula model for tracking and healing assessment. Herein, the thermoresponsive method was used to construct the sheets with cells previously labelled with SPIONs. This scaffold-free approach of magneto-fluorescent sheets enabled to acquire data on graft implantation and monitoring by MRI and by probe-based confocal 
laser endomicroscopy. Overall, EGF and VEGF were upregulated and anti-inflammatory factors, TGF- $\beta 2$ and IL-10, increased in comparison with control mice. This strategy evidenced theranostic potential of magnetic cell sheets for following-up therapeutic outcomes. $^{97}$

ii. Regeneration of tissues and organs with magnetically assisted technologies. The translation of TE strategies to the investigation of regenerative approaches is, most of the time, envisaged through the use of 3D structures, including hydrogels and scaffolds.

Hydrogels are characterized by a stable high-water content 3D network with biocompatible and biodegradable properties. This 3D microenvironment is quite efficient to be used for delivery purposes and provides a modifiable matrix for cell adhesion, migration, and/or differentiation. The hydrogel spectrum includes fully injectable to hard cross-linked matrices that can be tuned to respond to environmental changes in $\mathrm{pH}$, temperature, light, or magnetic forces to enable the controlled delivery of nanomedicines.

Magnetic hydrogels typically incorporate MNPs to render the matrix with magnetic responsiveness to improve the regeneration of multiple tissues. ${ }^{84}$ These include the simple incorporation of MNPs in the matrix ${ }^{98}$ embedding the nanoparticles or functionalizing MNPs to crosslink to the hydrogel mesh. ${ }^{84}$ Other strategies aim to re-organize matrix and cells into aligned architectures ${ }^{99,100}$ that may favour ordered deposition of collagenous matrix by the cells. This could be particularly promising for tissues characterized by an ECM formed by collagen fibers units with specific architecture/alignment, including cardiac, nervous, and musculoskeletal tissues such as tendons.

For example, Zwi-Dantsis et al. ${ }^{85}$ used antibody functionalized SPIONs to target and label human induced pluripotent-stem-cellderived cardiomyocytes (hiPSC-CMs). The cells, incorporated in collagen hydrogels, were exposed to different magnetic fields (ring, two parallel magnets, circular magnet) and aligned along the direction of the MF during the gelation period, thus creating different patterned cardiac tissue constructs. The dynamic manipulation of magnetically labelled cells within 3D biomaterials holds potential to be applied for the design of different microstructure orientations for TE therapeutic applications.

In tissue interfaces it is fundamental to design versatile systems to address the requirements of different tissues. A magnetic carrier system was developed to accommodate a chondroitin sulfate matrix enriched with platelet lysate with GFs delivery and cell carrier functionalities aimed to engineer tendon-bone interface. ${ }^{84}$ The system was composed of two interconnected units (bone and tendon units) that enabled spatial distribution, proliferation and differentiation of pre-osteoblasts and human tendon cells and in which MF impacted cell morphology and cell production of a tendon- and bone-like ECM. ${ }^{84}$

Another example is the 3D collagen hydrogel developed by Antman-Passig and Shefi to promote neuronal regeneration. ${ }^{99}$ SPIONs were applied to form strings aligned along the direction of MF lines which led to the alignment of collagen fibers. This structure also led to the induced alignment and growth of cultured neurons in the direction of the magnetic cues. The ability to inject the hydrogel into an injured site that can be remotely orientated through an EMF, holds potential to fill defects and proceed to the tissue substitute alignment afterwards according to the direction of tissue ECM and opens future possibilities for therapeutic engineered scaffolds.

In the last decade, the fabrication of scaffolds with magnetic properties has been mostly targeted to musculoskeletal TE, especially for bone tissue, being recently reviewed by Xia and colleagues. ${ }^{101}$ Strategies applying magnetic responsive scaffolds combined with magnetic actuation have shown positive outcomes in bone ${ }^{102,103}$ tendon, ${ }^{104-106}$ cardiac, ${ }^{107}$ cartilage, ${ }^{108}$ and vascular ${ }^{109}$ TE. The concept behind relies on the instructive and responsiveness features of the magnetic biomaterial to be implanted. ${ }^{110}$ Degradable polymeric-based biomaterials (synthetic and naturally derived polymers) have been the most adopted as the base for the incorporation of MNPs.

Goranov and co-workers ${ }^{103}$ magnetically labelled mesenchymal stem cells and human umbilical vein endothelial cells (HUVECs), and aligned them on the opposite sides of the fibers of a $3 \mathrm{D}$ magnetic scaffold (made of Fe-doped hydroxyapatite (FeHA) and PCL) under an EMF. This 3D implantable scaffold was developed to reconstruct the bone microarchitecture, especially the vascularization of the engineered bone through magnetic manipulation of osteogenic and vasculogenic cells.

Over the past few years, our group has been developing magnetic scaffolds for tendon TE applications. As pioneering work, a 3D printing technology was used to fabricate an aligned fibrous structure of starch and polycaprolactone (SPCL) dopped with SPIONs. ${ }^{106}$ This work showed promise outcomes in the commitment of human adipose stem cells towards tenogenesis and has been recently taken forward heading to a mechanostimulation platform in mediating mechanotransduction effects through TGF- $\beta / \operatorname{Smad} 2 / 3$ signalling. ${ }^{111}$

Other fiber production methods as electrospinning have been pursued in advancing magnetically responsive scaffolds. Magnetic yarns of continuous and aligned electrospun threads of PCL and cellulose nanocrystals (CNCs) have been recently reported by us to activate $\mathrm{YAP} / \mathrm{TAZ}$ signalling in response to magnetic stimulus. ${ }^{104}$ The magneto mechanical stimulation has led to marked upregulation of genes and increased protein levels associated to tendon tissues, suggesting a synergistic effect of topography and mechanical actuation on the tenogenic commitment. ${ }^{104}$

Magnetic electrospun fiber multilayered composites have been also designed as nerve guidance conduit (NGC) for promoting nerve regeneration. ${ }^{83}$ The outer and inner layers were made of PCL and PCL loaded with melatonin (MLT), respectively. In the middle layer PCL was loaded with SPIONs. In the presence of these conduits, macrophage phenotype switched from M1 to M2 that facilitates nerve regrowth. The CD68 and IL-6 expression was significantly reduced while the CD206 and IL-10 expression notably increased in comparison with the control groups. The multilayered NGC promoted functional improvement and electrophysiological recovery of regenerated sciatic nerves in vivo with inhibited oxidative stress and inflammation. Another example of magnetic responsive biomaterials in the form of membranes have been also developed 
by us, showing to be biocompatible and to positively influence inflammation responses in a rat ectopic model. ${ }^{112}$ These SPIONs impregnated SPCL based membranes (magSPCL) were also approached for the applicability of magnetic actuated therapies for tendon healing in an inflammatory context. ${ }^{105}$ In this work, the gene expression of pro-inflammatory cytokines and MMPs decreased in IL-1 $\beta$ primed tendon derived cells cultured onto magSPCL membranes under PEMF, while the expression of TIMP-1 and anti-inflammatory genes was shown to increase. PEMF antagonized the effect of IL-1 $\beta$ treatment on these cells, resulting in a decrease in the expression of $\mathrm{NF}-\mathrm{k} \beta$ and proinflammatory mediators. ${ }^{105}$ Interestingly, M2 type macrophages were only detected on PEMF actuated magSPCL membranes, which highlights the potential of magnetic responsive biomaterials for modulating inflammation and prospecting tissue regeneration.

\section{Conclusions and future perspectives}

In recent years, we have witnessed an accelerated growth in applying magnetic tools for biological and biomedical applications with the advent of nanoscale precise and evolving tools pushing the detection limit to early stage disease diagnosis, and to finely guided cell and tissue oriented treatments. However, there are hurdles to be overcome. For instance, in avoiding aggregation and achieving high magnetic moments, and on scheduling MNPs permanence inside the body to exert a particular role. Nevertheless, the limited knowledge motivates the development for improved and highly controlled synthesis procedures, new routes of administration and in vivo models to confirm stability, targeting and biological responses to magnetic based systems. It is though, undisputable that the versatility of MNPs as screening and diagnosis tools would disclose unprecedented contributions to the molecular and cellular biology fields. MNPs can improve high fidelity and efficient techniques as PCR and MRI, detect and quantify individual cells in living organisms, and ultimately gather more relevant information to better predict patients' needs. Simultaneously, MNPs can also accomplish specific targeting of tissues and cell subsets in human diseases providing a non-invasive approach to screen and guide abnormal cell responses, define treatment options and monitoring responses to therapies. The control of abnormal processes and the guidance of cell responses to desirable outcomes may also contribute for improving aging and for the management of comorbidities as some diseases are stimulated by persistent abnormal cues, especially at later stages in life. The paradigm is now focused on the new possibilities for tissue and organ regeneration. The increasing sensitivity and contrast of MNPs will enable a faster, more accurate and earlier identification of the signs of pathology. The combination of these features with precise actuation of magnetic-based therapies will enable the setup of theranostic platforms articulating different pathophysiology players (e.g. instructive molecules, cell guidance, niche contributions) in an integrated perspective to successfully improve the regenerative potential of injured tissues, to find new treatment regimens, and to prevent the progression of diseases and of chronic disorders.

\section{Conflicts of interest}

There are no conflicts of interest to declare.

\section{Acknowledgements}

This research was funded by the ERC CoG grant MagTendon (No. 772817), H2020 Twinning project Achilles (No. 810850), FCT Project MagTT PTDC/CTM-CTM/29930/2017 (POCI-01-0145-FEDER29930), and project Norte-01-0145-FEDER-02219015 supported by Norte Portugal Regional Operational Programme (NORTE 2020). Ana F. Almeida is funded by Fundação para a Ciência e Tecnologia (FCT) - Doctoral Grant SFRD/BD/144816/2019.

\section{Notes and references}

1 M. W. Freeman, A. Arrott and J. H. L. Watson, J. Appl. Phys., 1960, 31, S404-S405.

2 H. Shokrollahi, A. Khorramdin and G. Isapour, J. Magn. Magn. Mater., 2014, 369, 176-183.

3 S. Bisso and J.-C. Leroux, Int. J. Pharm., 2020, 578, 119098. 4 I. Perçin, V. Karakoç, S. Akgöl, E. Aksöz and A. Denizli, Mater. Sci. Eng., C, 2012, 32, 1133-1140.

5 A. I. Gonçalves, D. Berdecka, M. T. Rodrigues, A. D. Eren, J. de Boer, R. L. Reis and M. E. Gomes, J. Tissue Eng. Regener. Med., 2019, 13, 2204-2217.

6 A. I. Gonçalves, P. M. Gershovich, M. T. Rodrigues, R. L. Reis and M. E. Gomes, J. Tissue Eng. Regener. Med., 2018, 12, 762-774.

7 M. Cruz-Acuña, J. R. Halman, K. A. Afonin, J. Dobson and C. Rinaldi, Nanoscale, 2018, 10, 17761-17770.

8 H. Zhu, L. Zhang, S. Tong, C. M. Lee, H. Deshmukh and G. Bao, Nat. Biomed. Eng., 2019, 3, 126-136.

9 G. Mohammadi Ziarani, M. Malmir, N. Lashgari and A. Badiei, RSC Adv., 2019, 9, 25094-25106.

10 T. Vangijzegem, D. Stanicki and S. Laurent, Expert Opin. Drug Delivery, 2019, 16, 69-78.

11 D. Kami, S. Takeda, Y. Itakura, S. Gojo, M. Watanabe and M. Toyoda, Int. J. Mol. Sci., 2011, 12, 3705-3722.

12 M. S. Miranda, M. T. Rodrigues, R. M. A. Domingues, R. R. Costa, E. Paz, C. Rodríguez-Abreu, P. Freitas, B. G. Almeida, M. A. Carvalho, C. Gonçalves, C. M. Ferreira, E. Torrado, R. L. Reis, J. Pedrosa and M. E. Gomes, Adv. Healthcare Mater., 2018, 7, 1800124.

13 B. Chalidis, N. Sachinis, A. Assiotis, G. Maccauro and F. Graziani, Int. J. Immunopathol. Pharmacol., 2011, 24, 17-20.

14 L. Maurizi, A. L. Papa, L. Dumont, F. Bouyer, P. Walker, D. Vandroux and N. Millot, J. Biomed. Nanotechnol., 2015, 11, 126-136.

15 T. A. Wynn and K. M. Vannella, Immunity, 2016, 44, 450-462.

16 R. M. Stanbury and E. M. Graham, Br. J. Ophthalmol., 1998, 82, 704-708.

17 G. M. Anstead, Adv. Wound Care, 1998, 11, 277-285. 
18 N. X. Landén, D. Li and M. Ståhle, Cell. Mol. Life Sci., 2016, 73, 3861-3885.

19 L. Ferroni, C. Gardin, O. Dolkart, M. Salai, S. Barak, A. Piattelli, H. Amir-Barak and B. Zavan, Sci. Rep., 2018, 8, 5108.

20 A. Vinhas, A. F. Almeida, A. I. Goncalves, M. T. Rodrigues and M. E. Gomes, Int. J. Mol. Sci., 2020, 21, 5441.

21 J. Wosik, W. Chen, K. Qin, R. M. Ghobrial, J. Z. Kubiak and M. Kloc, Biophys. J., 2018, 114, 2001-2013.

22 M. Rotherham and A. J. E. Haj, PLoS One, 2015, 10, e0121761.

23 J. R. Henstock, M. Rotherham and A. J. E. Haj, J. Tissue Eng., 2018, 9, 2041731418808695.

24 M. Rotherham, J. R. Henstock, O. Qutachi and A. J. E. Haj, Nanomedicine, 2018, 14, 173-184.

25 C. L. Ross, Y. Zhou, C. E. McCall, S. Soker and T. L. Criswell, Bioelectricity, 2019, 1, 247-259.

26 A. I. Gonçalves, M. Rotherham, H. Markides, M. T. Rodrigues, R. L. Reis, M. E. Gomes and A. J. E. Haj, Nanomedicine, 2018, 14, 1149-1159.

27 A. Vinhas, M. T. Rodrigues, A. I. Gonçalves, R. L. Reis and M. E. Gomes, J. Orthop. Res., 2020, 38, 160-172.

28 A. Prasad, D. B. L. Teh, A. Blasiak, C. Chai, Y. Wu, P. M. Gharibani, I. H. Yang, T. T. Phan, K. L. Lim, H. Yang, X. Liu and A. H. All, Sci. Rep., 2017, 7, 6743.

29 S. Gordon and F. O. Martinez, Immunity, 2010, 32, 593-604.

30 A. Sica and A. Mantovani, J. Clin. Invest., 2012, 122, 787-795.

31 C. Guo and L. J. Kaufman, Biomaterials, 2007, 28, 1105-1114.

32 J. Kim and K. Tanner, Curr. Protoc. Cell Biol., 2016, 70, 25.3.1-25.3.14.

33 J. Kudr, Y. Haddad, L. Richtera, Z. Heger, M. Cernak, V. Adam and O. Zitka, Nanomaterials, 2017, 7, 243.

34 E. K. Schlachter, H. R. Widmer, A. Bregy, T. LönnforsWeitzel, I. Vajtai, N. Corazza, V. J. P. Bernau, T. Weitzel, P. Mordasini, J. Slotboom, G. Herrmann, S. Bogni, H. Hofmann, M. Frenz and M. Reinert, Int. J. Nanomed., 2011, 6, 1793-1800.

35 N. Singh, G. J. S. Jenkins, R. Asadi and S. H. Doak, Nano Rev., 2010, 1, 5358.

36 M. Mahmoudi, S. Sant, B. Wang, S. Laurent and T. Sen, Adv. Drug Delivery Rev., 2011, 63, 24-46.

37 S. Venkateswarlu, Y. S. Rao, T. Balaji, B. Prathima and N. V. V. Jyothi, Mater. Lett., 2013, 100, 241-244.

38 K. Kumar, A. M. Nightingale, S. H. Krishnadasan, N. Kamaly, M. Wylenzinska-Arridge, K. Zeissler, W. R. Branford, E. Ware, A. J. deMello and J. C. deMello, J. Mater. Chem., 2012, 22, 4704-4708.

39 J. Mosayebi, M. Kiyasatfar and S. Laurent, Adv. Healthcare Mater., 2017, 6, 1700306.

40 S. M. Dadfar, K. Roemhild, N. I. Drude, S. von Stillfried, R. Knüchel, F. Kiessling and T. Lammers, Adv. Drug Delivery Rev., 2019, 138, 302-325.

41 S. Palanisamy and Y.-M. Wang, Dalton Trans., 2019, 48, 9490-9515.

42 M. Mahmoudi, A. Simchi, A. S. Milani and P. Stroeve, J. Colloid Interface Sci., 2009, 336, 510-518.
43 L. Yue, C. Sun, C. H. T. Kwong and R. Wang, J. Mater. Chem. B, 2020, 8, 2749-2753.

44 F. Benyettou, I. Milosevic, Y. Lalatonne, F. Warmont, R. Assah, J.-C. Olsen, M. Jouaid, L. Motte, C. Platas-Iglesias and A. Trabolsi, J. Mater. Chem. B, 2013, 1, 5076-5082.

45 T. Guo, M. Lin, J. Huang, C. Zhou, W. Tian, H. Yu, X. Jiang, J. Ye, Y. Shi, Y. Xiao, X. Bian and X. Feng, J. Nanomater., 2018, 2018, 7805147.

46 Y. C. Park, J. B. Smith, T. Pham, R. D. Whitaker, C. A. Sucato, J. A. Hamilton, E. Bartolak-Suki and J. Y. Wong, Colloids Surf., B, 2014, 119, 106-114.

47 Q. Feng, Y. Liu, J. Huang, K. Chen, J. Huang and K. Xiao, Sci. Rep., 2018, 8, 2082.

48 C. Schmidtke, R. Eggers, R. Zierold, A. Feld, H. Kloust, C. Wolter, J. Ostermann, J.-P. Merkl, T. Schotten, K. Nielsch and H. Weller, Langmuir, 2014, 30, 11190-11196.

49 K. Yang, Y. Liu, Y. Liu, Q. Zhang, C. Kong, C. Yi, Z. Zhou, Z. Wang, G. Zhang, Y. Zhang, N. M. Khashab, X. Chen and Z. Nie, J. Am. Chem. Soc., 2018, 140, 4666-4677.

50 E. J. Ngen, L. Wang, Y. Kato, B. Krishnamachary, W. Zhu, N. Gandhi, B. Smith, M. Armour, J. Wong, K. Gabrielson and D. Artemov, Sci. Rep., 2015, 5, 13628.

51 Y. Li, H. Wu, B. Ji, W. Qian, S. Xia, L. Wang, Y. Xu, J. Chen, L. Yang and H. Mao, ACS Appl. Bio Mater., 2020, 3, 4335-4347.

52 L. C. Wu, Y. Zhang, G. Steinberg, H. Qu, S. Huang, M. Cheng, T. Bliss, F. Du, J. Rao, G. Song, L. Pisani, T. Doyle, S. Conolly, K. Krishnan, G. Grant and M. Wintermark, Am. J. Neuroradiol., 2019, 40, 206-212.

53 P. Ludewig, N. Gdaniec, J. Sedlacik, N. D. Forkert, P. Szwargulski, M. Graeser, G. Adam, M. G. Kaul, K. M. Krishnan, R. M. Ferguson, A. P. Khandhar, P. Walczak, J. Fiehler, G. Thomalla, C. Gerloff, T. Knopp and T. Magnus, ACS Nano, 2017, 11, 10480-10488.

54 M. Graeser, F. Thieben, P. Szwargulski, F. Werner, N. Gdaniec, M. Boberg, F. Griese, M. Möddel, P. Ludewig, D. van de Ven, O. M. Weber, O. Woywode, B. Gleich and T. Knopp, Nat. Commun., 2019, 10, 1936.

55 Y. Xianyu, Q. Wang and Y. Chen, TrAC, Trends Anal. Chem., 2018, 106, 213-224.

56 C. Phurimsak, M. D. Tarn, S. A. Peyman, J. Greenman and N. Pamme, Anal. Chem., 2014, 86, 10552-10559.

57 M. Pohanka, Talanta, 2018, 178, 970-973.

58 Y. Li, B. Srinivasan, Y. Jing, X. Yao, M. A. Hugger, J.-P. Wang and C. Xing, J. Am. Chem. Soc., 2010, 132, 4388-4392.

59 Y. Pang, C. Wang, J. Wang, Z. Sun, R. Xiao and S. Wang, Biosens. Bioelectron., 2016, 79, 574-580.

60 J. Wang, Z. Ali, J. Si, N. Wang, N. He and Z. Li, J. Nanosci. Nanotechnol., 2017, 17, 802-806.

61 S. Jhunjhunwala and S. R. Little, Cell Cycle, 2011, 10, 2047-2048.

62 T. Kitagawa, H. Kosuge, M. Uchida, Y. Iida, R. L. Dalman, T. Douglas and M. V. McConnell, J. Magn. Reson. Imaging, 2017, 45, 1144-1153.

63 A. Al Faraj, A. S. Shaik, S. Afzal, B. Al Sayed and R. Halwani, Int. J. Nanomed., 2014, 9, 1491-1503. 
64 Y. Kono, S. Gogatsubo, T. Ohba and T. Fujita, Drug Delivery, 2019, 26, 935-943.

65 H. Kang, H. J. Jung, S. K. Kim, D. S. H. Wong, S. Lin, G. Li, V. P. Dravid and L. Bian, ACS Nano, 2018, 12, 5978-5994.

66 L. J. Santos, R. L. Reis and M. E. Gomes, Trends Biotechnol., 2015, 33, 471-479.

67 T. Iskratsch, H. Wolfenson and M. P. Sheetz, Nat. Rev. Mol. Cell Biol., 2014, 15, 825-833.

68 M. Wall, D. Butler, A. E. Haj, J. C. Bodle, E. G. Loboa and A. J. Banes, J. Orthop. Res., 2017, 36, 605-619.

69 A. M. Matos, A. I. Gonçalves, A. J. E. Haj and M. E. Gomes, Nanoscale Adv., 2020, 2, 140-148.

70 J. Dobson, S. H. Cartmell, A. Keramane and A. J. El Haj, IEEE Trans Nanobioscience, 2006, 5, 173-177.

71 S. Hughes, S. McBain, J. Dobson and A. J. El Haj, J. R. Soc., Interface, 2008, 5, 855-863.

72 J. R. Henstock, M. Rotherham, H. Rashidi, K. M. Shakesheff and A. J. El Haj, Stem Cells Transl. Med., 2014, 3, 1363-1374.

73 P.-C. Wu, D.-B. Shieh, H.-T. Hsiao, J. C.-F. Wang, Y.-C. Lin and Y.-C. Liu, J. Nanobiotechnol., 2018, 16, 49.

74 M. Nardoni, E. Della Valle, M. Liberti, M. Relucenti, M. A. Casadei, P. Paolicelli, F. Apollonio and S. Petralito, Nanomaterials, 2018, 8, 196.

75 K. Kunath, A. von Harpe, D. Fischer, H. Petersen, U. Bickel, K. Voigt and T. Kissel, J. Controlled Release, 2003, 89, 113-125.

76 Y. Cao, Y. F. Tan, Y. S. Wong, M. W. J. Liew and S. Venkatraman, Mar. Drugs, 2019, 17, 381.

77 H. Zhu, L. Zhang, S. Tong, C. M. Lee, H. Deshmukh and G. Bao, Nat. Biomed. Eng., 2019, 3, 126-136.

78 P. Wu, H. Chen, R. Jin, T. Weng, J. K. Ho, C. You, L. Zhang, X. Wang and C. Han, J. Transl. Med., 2018, 16, 29.

79 M. Hryhorowicz, B. Grześkowiak, N. Mazurkiewicz, P. Śledziński, D. Lipiński and R. Słomski, Mol. Biotechnol., 2019, 61, 173-180.

80 S. S. Rohiwal, N. Dvorakova, J. Klima, M. Vaskovicova, F. Senigl, M. Slouf, E. Pavlova, P. Stepanek, D. Babuka, H. Benes, Z. Ellederova and K. Stieger, Sci. Rep., 2020, 10, 4619.

81 H. Skaat, O. Ziv-Polat, A. Shahar, D. Last, Y. Mardor and S. Margel, Adv. Healthcare Mater., 2012, 1, 168-171.

82 A. I. Gonçalves, M. T. Rodrigues and M. E. Gomes, Acta Biomater., 2017, 63, 110-122.

83 X. Chen, X. Ge, Y. Qian, H. Tang, J. Song, X. Qu, B. Yue and W.-E. Yuan, Adv. Funct. Mater., 2020, 30, 2004537.

84 E. D. Silva, P. S. Babo, R. Costa-Almeida, R. M. A. Domingues, B. B. Mendes, E. Paz, P. Freitas, M. T. Rodrigues, P. L. Granja and M. E. Gomes, Nanomedicine, 2018, 14, 2375-2385.

85 L. Zwi-Dantsis, B. Wang, C. Marijon, S. Zonetti, A. Ferrini, L. Massi, D. J. Stuckey, C. M. Terracciano and M. M. Stevens, Adv. Mater., 2020, 32, 1904598.

86 A. I. Goncalves, M. S. Miranda, M. T. Rodrigues, R. L. Reis and M. E. Gomes, Biomed. Mater., 2018, 13, 054001.

87 A. Ito, M. Hayashida, H. Honda, K. Hata, H. Kagami, M. Ueda and T. Kobayashi, Tissue Eng., 2004, 10, 873-880.

88 T. Kito, R. Shibata, M. Ishii, H. Suzuki, T. Himeno, Y. Kataoka, Y. Yamamura, T. Yamamoto, N. Nishio, S. Ito,
Y. Numaguchi, T. Tanigawa, J. K. Yamashita, N. Ouchi, H. Honda, K. Isobe and T. Murohara, Sci. Rep., 2013, 3, 1418.

89 M. Ishii, R. Shibata, Y. Numaguchi, T. Kito, H. Suzuki, K. Shimizu, A. Ito, H. Honda and T. Murohara, Arterioscler., Thromb., Vasc. Biol., 2011, 31, 2210-2215.

90 H. Akiyama, A. Ito, Y. Kawabe and M. Kamihira, Biomaterials, 2010, 31, 1251-1259.

91 K. Shimizu, A. Ito, T. Yoshida, Y. Yamada, M. Ueda and H. Honda, J. Biomed. Mater. Res., Part B, 2007, 82, 471-480.

92 Y. Yamamoto, A. Ito, M. Kato, Y. Kawabe, K. Shimizu, H. Fujita, E. Nagamori and M. Kamihira, J. Biosci. Bioeng., 2009, 108, 538-543.

93 Y. Yamamoto, A. Ito, H. Fujita, E. Nagamori, Y. Kawabe and M. Kamihira, Tissue Eng., Part A, 2011, 17, 107-114.

94 H. Akiyama, A. Ito, M. Sato, Y. Kawabe and M. Kamihira, Int. J. Mol. Sci., 2010, 11, 2910-2920.

95 A. Vinhas, M. T. Rodrigues and M. E. Gomes, in Cell Biology and Translational Medicine, Volume 2: Approaches for Diverse Diseases and Conditions, ed. K. Turksen, Springer International Publishing, Cham, 2018, DOI: 10.1007/5584_2018_258, pp. 37-46.

96 W. Zhang, G. Yang, X. Wang, L. Jiang, F. Jiang, G. Li, Z. Zhang and X. Jiang, Adv. Mater., 2017, 29.

97 G. Rahmi, L. Pidial, A. K. Silva, E. Blondiaux, B. Meresse, F. Gazeau, G. Autret, D. Balvay, C. A. Cuenod, S. Perretta, B. Tavitian, C. Wilhelm, C. Cellier and O. Clement, Theranostics, 2016, 6, 739-751.

98 E. G. Popa, V. E. Santo, M. T. Rodrigues and M. E. Gomes, Polymers, 2016, 8, 28.

99 M. Antman-Passig and O. Shefi, Nano Lett., 2016, 16, 2567-2573.

100 S. Araújo-Custódio, M. Gomez-Florit, A. R. Tomás, B. B. Mendes, P. S. Babo, S. M. Mithieux, A. Weiss, R. M. A. Domingues, R. L. Reis and M. E. Gomes, ACS Biomater. Sci. Eng., 2019, 5, 1392-1404.

101 Y. Xia, J. Sun, L. Zhao, F. Zhang, X. J. Liang, Y. Guo, M. D. Weir, M. A. Reynolds, N. Gu and H. H. K. Xu, Biomaterials, 2018, 183, 151-170.

102 J. Meng, B. Xiao, Y. Zhang, J. Liu, H. Xue, J. Lei, H. Kong, Y. Huang, Z. Jin, N. Gu and H. Xu, Sci. Rep., 2013, 3, 2655.

103 V. Goranov, T. Shelyakova, R. De Santis, Y. Haranava, A. Makhaniok, A. Gloria, A. Tampieri, A. Russo, E. Kon, M. Marcacci, L. Ambrosio and V. A. Dediu, Sci. Rep., 2020, 10, 2289.

104 A. R. Tomás, A. I. Gonçalves, E. Paz, P. Freitas, R. M. A. Domingues and M. E. Gomes, Nanoscale, 2019, 11, 18255-18271.

105 A. Vinhas, M. T. Rodrigues, A. I. Gonçalves, R. L. Reis and M. E. Gomes, Acta Biomater., 2020, 117, 235-245.

106 A. I. Gonçalves, M. T. Rodrigues, P. P. Carvalho, M. BañobreLópez, E. Paz, P. Freitas and M. E. Gomes, Adv. Healthcare Mater., 2016, 5, 213-222.

107 Y. Sapir, B. Polyak and S. Cohen, Nanotechnology, 2014, 25, 014009. 
108 C. Zhang, Y. Z. Cai, X. J. Lin and Y. Wang, Front. Cell Dev. Biol., 2020, 8, 526.

109 Y. Sapir, S. Cohen, G. Friedman and B. Polyak, Biomaterials, 2012, 33, 4100-4109.

110 A. I. Gonçalves, M. S. Miranda, M. T. Rodrigues, R. L. Reis and M. E. Gomes, Biomed. Mater., 2018, 13, 054001.
111 A. M. Matos, A. I. Gonçalves, M. T. Rodrigues, M. S. Miranda, A. J. E. Haj, R. L. Reis and M. E. Gomes, Acta Biomater., 2020, 113, 488-500.

112 L. Santos, M. Silva, A. I. Gonçalves, T. Pesqueira, M. T. Rodrigues and M. E. Gomes, Nanomedicine, 2016, 11, 1107-1122. 